\title{
COVID-19 Monoclonal Antibody Infusions: A Multidisciplinary Initiative to Operationalize EUA Novel Treatment Options
}

\author{
Kathleen Jodoin, PharmD, BCPS, David Farcy, MD, Ana Caldera, PharmD, Michael Dalley, DO, \\ Brijesh Patel, MD, Wendy Stuart, RN, Gustavo Tejas, RN, Michael Atisha, RN, \\ Tarang Kheradia, MBBS, Erich Glasbrenner, RN, and Robert Goldszer, MD, MBA
}

\section{ABSTRACT}

Objective: To develop and implement a process for administering COVID-19 monoclonal antibody infusions for outpatients with mild or moderate COVID-19 at high risk for hospitalization, using multidisciplinary collaboration, US Food and Drug Administration (FDA) guidance, and infection prevention standards.

Methods: When monoclonal antibody therapy became available for mild or moderate COVID-19 outpatients via Emergency Use Authorization (EUA), our institution sought to provide this therapy option to our patients. We describe the process for planning, implementing, and maintaining a successful program for administering novel therapies based on FDA guidance and infection prevention standards. Keys components of our implementation process were multidisciplinary planning involving decision makers and stakeholders; setting realistic goals in the process; team communication; and measuring and reporting quality improvement on a regular basis.

Results: A total of 790 COVID-19 monoclonal antibody infusions were administered from November 20, 2020 to March 5, 2021. Steps to minimize the likelihood of adverse drug reactions were implemented and a low incidence $(<$ $1 \%)$ has occurred. There has been no concern from staff regarding infection during the process. Rarely, patients have raised cost-related concerns, typically due to incomplete communication regarding billing prior to the infusion. Patients, families, nursing staff, physicians, pharmacy, and hospital administration have expressed satisfaction with the program.

Conclusion: This process can provide a template for other hospitals or health care delivery facilities to provide novel therapies to patients with mild or moderate COVID-19 in a safe and effective manner.

Keywords: COVID-19; monoclonal antibody; infusion; emergency use authorization.

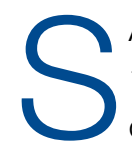

ARS-CoV-2 and the disease it causes, COVID19 , have transformed from scientific vernacular to common household terms. It began with a cluster of pneumonia cases of unknown etiology in December 2019 in Wuhan, China, with physicians there reporting a novel coronavirus strain (2019-nCoV), now referred to as SARS-CoV-2. Rapid spread of this virus resulted in the World Health Organization (WHO) declaring an international public health emergency. Since this time, the virus has evolved into a worldwide pandemic. COVID19 has dramatically impacted our society, resulting in more than 2.63 million global deaths as of this writing, of which more than 527,000 deaths have occurred in the United States. ${ }^{1}$ This novel virus has resulted in a flurry of literature, research, therapies, and collaboration across multiple disciplines in an effort to prevent, treat, and mitigate cases and complications of this disease.

On November 9, 2020, and November 21, 2020, the US Food and Drug Administration (FDA) issued Emergency Use Authorizations (EUA) for 2 novel COVID19 monoclonal therapies, bamlanivimab2-3 and casirivimab/imdevimab, ${ }^{3-4}$ respectively. The EUAs granted

From Mount Sinai Medical Center, Miami Beach, FL. 
permission for these therapies to be administered for the treatment of mild to moderate COVID-19 in adult and pediatric patients ( $\geq 12$ years and weighing at least $40 \mathrm{~kg}$ ) with positive results of direct SARS-CoV-2 viral testing and who are at high risk for progressing to severe COVID-19 and/or hospitalization. The therapies work by targeting the SARS-CoV-2 spike protein and subsequent attachment to human angiotensin-converting enzyme 2 receptors. Clinical trial data leading to the EUA demonstrated a reduction in viral load, safe outcome, and most importantly, fewer hospitalization and emergency room visits, as compared to the placebo group..$^{5-7}$ The use of monoclonal antibodies is not new and gained recognition during the Ebola crisis, when the monoclonal antibody to the Ebola virus showed a significant survival benefit. ${ }^{8}$ Providing monoclonal antibody therapy soon after symptom onset aligns with a shift from the onset of the pandemic to the current focus on the administration of pharmaceutical therapy early in the disease course. This shift prevents progression to severe COVID-19, with the goal of reducing patient mortality, hospitalizations, and strain on health care systems.

The availability of novel neutralizing monoclonal antibodies for COVID-19 led to discussions of how to incorporate these therapies as new options for patients. Our institution networked with colleagues from multiple disciplines to discuss processes and policies for the safe administration of the monoclonal antibody infusion therapies. Federal health leaders urge more use of monoclonal antibodies, but many hospitals have been unable to successfully implement infusions due to staff and logistical challenges. ${ }^{9}$ This article presents a viable process that hospitals can use to provide these novel therapies to outpatients with mild to moderate COVID-19.

\section{The Mount Sinai Medical Center, Florida Experience}

Mount Sinai Medical Center in Miami Beach, Florida, is the largest private, independent, not-for-profit teaching hospital in South Florida, comprising 672 licensed beds and supporting 150,000 emergency department (ED) visits annually. Per the EUA criteria for use, COVID-19 monoclonal antibody therapies are not authorized for patients who are hospitalized or who require oxygen therapy due to COVID-19. Therefore, options for outpatient administration needed to be evaluated. Directly following the first EUA press release, a task force of key stakeholders was assembled to brainstorm and develop a process to offer this therapy to the community. A multidisciplinary task force with representation from the ED, nursing, primary care, hospital medicine, pharmacy, risk management, billing, information technology, infection prevention, and senior level leadership participated (Table).

The task force reviewed institutional outpatient locations to determine whether offering this service would be feasible (eg, ED, ambulatory care facilities, cancer center). The ED was selected because it would offer the largest array of appointment times to meet the community needs with around-the-clock availability. While Mount Sinai Medical Center offers care in 3 emergency center locations in Aventura, Hialeah, and Miami Beach, it was determined to initiate the infusions at the main campus center in Miami Beach only. The main campus affords an onsite pharmacy with suitable staffing to prepare the anticipated volume of infusions in a timely manner, as both therapies have short stabilities following preparation. Thus, it was decided that patients from freestanding emergency centers in Aventura and Hialeah would be moved to the Miami Beach ED location to receive therapy. Operating at a single site also allowed for more rapid implementation, monitoring, and ability to make modifications more easily. Discussions for the possible expansion of COVID-19 monoclonal antibody infusions at satellite locations are underway.

On November 20, 2020, 11 days after the formation of the multidisciplinary task force, the first COVID-19 monoclonal infusion was successfully administered. Figure 1 depicts the timeline from assessment to program implementation. Critical to implementation was the involvement of decision makers from all necessary departments early in the planning process to ensure that standard operating procedures were followed and that the patients, community, and organization had a positive experience. This allowed for simultaneous planning of electronic health record (Epic; EHR) builds, departmental workflows, and staff education, as described in 


\begin{tabular}{ll}
\hline Table. List of Key Stakeholders and Responsibilities \\
\hline Department or position & Responsibility \\
\hline Chief Nursing Officer & Decision maker on go or no go, nursing involvement \\
\hline Chief Medical Officer & Decision maker on go or no go, physician involvement \\
\hline Director of Pharmacy & Decision maker on go or no go, pharmacy involvement \\
\hline Pharmacy Clinical Coordinator & Implementation process including preparation, delivery, patient safety and peer education \\
\hline Chair of Emergency Medicine & ED process and physician education and oversight \\
\hline Chief of General Medicine & Referring physician education and oversight \\
\hline Emergency Medicine Nursing & ED nursing staffing, education, safety and process \\
\hline Emergency Medicine Residents & ED process, learning, clinician input, peer education \\
\hline Infection Prevention & Infection prevention and education \\
\hline Information Technology & Medication order, order sets, documentation, interfaces as needed, and clinician education \\
\hline Patient Registration & Correctly and safely register and schedule all patients \\
\hline Revenue Cycle & Appropriate billing and assessment of expenses \\
\hline Security & Patient and staff safety \\
\hline ED, emergency department. &
\end{tabular}

the following section. Figure $\mathbf{2}$ shows the patient safety activities included in the implementation process.

\section{Key Stakeholder Involvement and Workflow}

On the day of bamlanivimab EUA release, email communication was shared among hospital leadership with details of the press release. Departments were quickly involved to initiate a task force to assess if and how this therapy could be offered at Mount Sinai Medical Center. The following sections explain the role of each stakeholder and their essential role to operationalize these novel EUA treatment options. The task force was organized and led by our chief medical officer and chief nursing officer.

\section{Information Technology}

Medication Ordering and Documentation EHR and Smart Pumps. Early in the pandemic, the antimicrobial stewardship (ASP) clinical coordinator became the designated point person for pharmacy assessment of novel COVID-19 therapies. As such, this pharmacist began reviewing the bamlanivimab and, later, the casirivimab/imdevimab EUA Fact Sheet for Health Care Providers. All necessary elements for the complete and safe ordering and dispensing of the medication were developed and reviewed by pharmacy administration and ED nursing leadership for input, prior to submitting to the information technology team for implementation. Building the COVID-19 monoclonal medication records into the EHR allowed for detailed direction (ie, administration and preparation instructions) to be consistently applied. The medication records were also built into hospital smart pumps so that nurses could access prepopulated, accurate volumes and infusion rates to minimize errors.

Order Set Development. The pharmacy medication build was added to a comprehensive order set (Figure 3), which was then developed to guide prescribers and standardize the process around ordering of COVID-19 monoclonal therapies. While these therapies are new, oncology monoclonal therapies are regularly administered to outpatients at Mount Sinai Cancer Center. The cancer center was therefore consulted on their process surrounding best practices in administration of monoclonal antibody therapies. This included protocols for medications used in pretreatment and management of hypersensitivity reactions and potential adverse drug reactions of both COVID-19 monoclonal therapies. These medication orders were selected by default in the order set to ensure that all patients received premedi- 


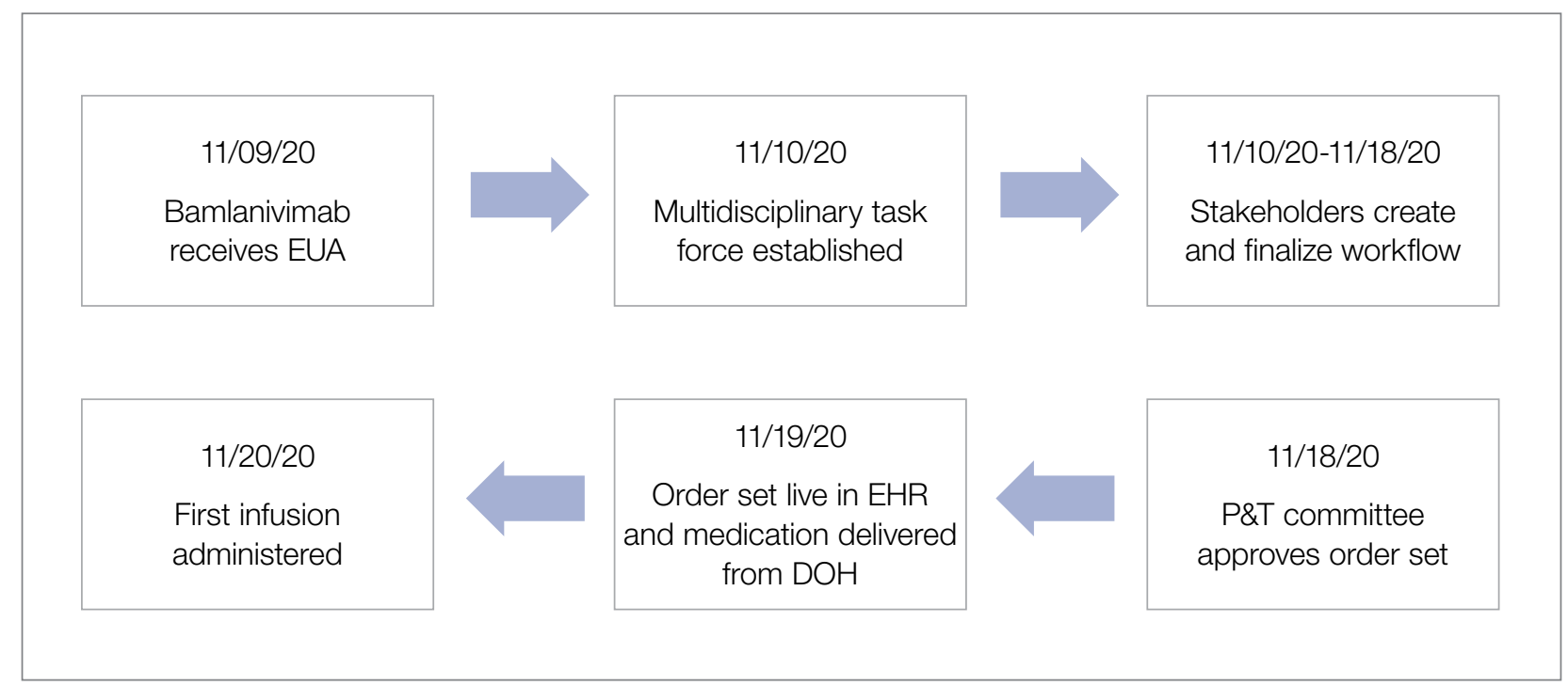

Figure 1. Process implementation timeline. EUA, Emergency Use Authorization; P\&T, Pharmacy and Therapeutics; DOH, department of health; EHR, electronic health record.

cations aimed at minimizing the risk of hypersensitivity reaction, and had as-needed medication orders, in the event a hypersensitivity reaction occurred. Reducing hypersensitivity reaction risk is important as well to increase the likelihood that the patient would receive full therapy, as management of this adverse drug reactions involves possible cessation of therapy depending on the level of severity. The pharmacy department also ensured these medications were stocked in ED automated dispensing cabinets to promote quick access. In addition to the aforementioned nursing orders, we added EUA criteria for use and hyperlinks to the Fact Sheets for Patients and Caregivers and Health Care Providers for each monoclonal therapy, and restricted ordering to ED physicians, nurse practitioners, and physician assistants.

The order set underwent multidisciplinary review by pharmacy administration, the chair of emergency medicine, physicians, and ED nursing leadership prior to presentation and approval by the Pharmacy and Therapeutics Committee. Lastly, at time of implementation, the order set was added to the ED preference list, preventing inpatient access. Additionally, as a patient safety action, freestanding orders of COVID-19 monoclonal therapies were disabled, so providers could only order therapies via the approved, comprehensive order set.
Preliminary Assessment Tool. A provider assessment tool was developed to document patient-specific EUA criteria for use during initial assessment (Figure 4). This tool serves as a checklist and is visible to the full multidisciplinary team in the patient's EHR. It is used as a resource at the time of pharmacist verification and ED physician assessment to ensure criteria for use are met.

\section{Outpatient Offices}

Patient Referral. Patients with symptoms or concerns of COVID-19 exposure can make physician appointments via telemedicine or in person at Mount Sinai Medical Center's primary care and specialty offices. At the time of patient encounter, physicians suspecting a COVID-19 diagnosis will refer patients for outpatient COVID-19 polymerase chain reaction (PCR) laboratory testing, which has an approximate 24-hour turnaround to results. Physicians also assess whether the patient meets EUA criteria for use, pending results of testing. In the event a patient meets EUA criteria for use, the physician provides patient counseling and requests verbal consent. Following this, the physician enters a note in the EHR describing the patient's condition, criteria for use evaluation, and the patient's verbal agreement to therapy. This preliminary screening is beneficial to begin planning with both the patient and ED to minimize 


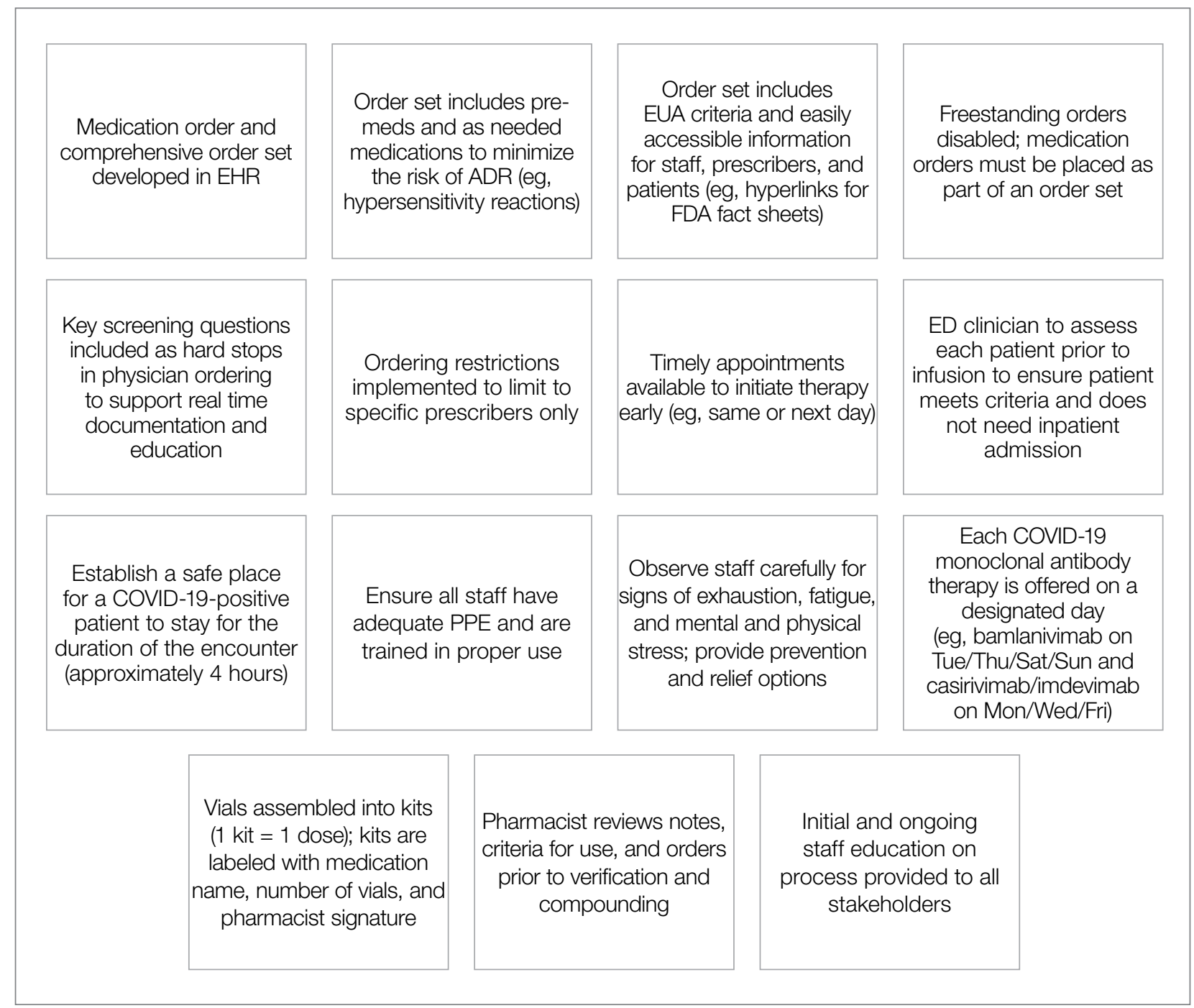

Figure 2. Important patient safety initiatives. ADR, adverse drug reaction; ED, emergency department; EHR, electronic health record; EUA, Emergency Use Authorization; FDA, US Food and Drug Administration; PPE, personal protective equipment.

delays. Patients are notified of the results of their test once available. If the COVID-19 PCR test returns positive, the physician will call the ED at the main campus and schedule the patient for COVID-19 monoclonal therapy. As the desired timeframe for administering COVID19 monoclonal therapies is within less than 10 days of symptom onset, timely scheduling of appointments is crucial. Infusion appointments are typically provided the same or next day. The patients are informed that they must bring documentation of their positive COVID-19 PCR test to their ED visit. Lastly, because patients are pretreated with medication that may potentially impair driving, they are instructed that they cannot drive themselves home; ride shares also are not allowed in order to limit the spread of infection.

\section{Emergency Department}

Patient Arrival and Screening. A COVID-19 patient can be evaluated in the ED 1 of 2 ways. The first option is via outpatient office referral, as described previously. Upon arrival to the ED, a second screening is performed to ensure the patient still meets EUA criteria for use and 
COVID MONOCLONAL IV THERAPY ORDER SET

Restricted to ED prescribers (includes ED attendings, ED nurse practitioners and ED Physician Assistants). Must obtain and document (1) patient consent and (2) provide Fact Sheet for Patients, Parents, and Caregivers prior to use (see links below).

Monitor for hypersensitivity including anaphylaxis and infusion-related reactions. If clinically significant hypersensitivity reaction or anaphylaxis occur, immediately discontinue administration and initiate appropriate medications and/or supportive therapy. The prescriber is responsible for mandatory reporting of all medication errors and serious adverse events within 7 calendar days via www.fda.gov/medwatch/report.htm. Reference for Fact Sheet for Healthcare Providers for full information.

\section{Exclusion Criteria:}

- Require inpatient admission

- Require oxygen therapy due to COVID-19

- Require an increase in baseline oxygen flow rate due to COVID-19 in those on chronic oxygen therapy due to underlying non-COVID-19 related comorbidity.

Inclusion Criteria:

- Mild to moderate COVID-19 and positive SARS-CoV-2 viral testing

- Adults and pediatric patients $\geq 12$ years of age and $240 \mathrm{~kg}$ who are at HIGH RISK for progressing to severe COVID.19 and/or hospitalization

- MUST meet AT LEAST ONE of the following HIGH RISK criteria

(1) $B M I \geq 35$

(2) Chronic Kidney Disease

(3) Diabetes

(4) Immunosuppressive disease or currently receiving immunosuppressive therapy

(5) $\geq 65$ years of age

(6) $\geq 55$ years of age AND have (a) cardiovascular disease, (b) HTN, OR (c) COPD/other chronic respiratory disease

(7) 12-17 years of age AND have (a) BMI $\geq 85$ th percentile for age and gender based on CDC growth charts, https $J /$ www.cdc.gov/growthcharts/clinical_charts.htm (b) sickle cell disease, (c) congenital or acquired heart disease, (d) neurodevelopmental disorders (e.g. cerebral palsy), (e) medical-related technological dependence (e.g. tracheostomy, gastrostomy, positive pressure ventilation not related to COVID-19), (f) asthma, reactive ainway or other chronic respiratory disease that requires daily medication for control

Bamlanivimab Fact Sheet for Patients. Parents, and Caregivers - ENGLISH

Bamlanivimab Fact Sheet for Patients, Parents, and Careqivers - SPANISH

Bamlanivimab Fact Sheet for Health Care Providers

Casirivimabsimdevimab Fact Sheet for Patients. Parents, and Caregivers - ENGUISH

Casirivimabfimdevimab Fact Sheet for Patients, Parents, and Careqivers - SPANISH

\section{Nursing Orders}

Nursing Orders [30411048]

[X] Treatment Conditions 1 MILD REACTIONS: (mild pruritis, flushing, rhinitis, rash, fever) IF PATIENT DEVELOPS RIGORS AND/OR CHILLS CONTACT MD IMMEDIATELY FOR ORDERS, CONSIDER DEMEROL $25 \mathrm{mg} I \mathrm{M} \times 1,1$. Stop infusion 2. Normal Saline at KVO 3. Diphenydramine $25 \mathrm{mg}$ IVP $\times 1$, then repeat every 3 hours prn for symptoms above 4 . Famotidine $20 \mathrm{mg}$ IV Push 5. Notify Physician 6 . Vital signs every 15 minutes until resolution of symptoms 7 . When symptoms resolve, resume infusion at $50 \%$ of the original rate. 8 . Complete ADR report.

[X] Treatment Conditions 2 MODERATE REACTIONS: (Generalizing pruritis, flushing, rash, back pain, dyspnea, SBP above $80 \mathrm{mmHg}$ ) IF PATIENT DEVELOPS RIGORS ANDIOR CHILLS CONTACT MD IMMEDIATELY FOR ORDERS. CONSIDER DEMEROL $25 \mathrm{ma} \mathrm{IM} \times 1$. 1. Stop Infusion 2. Have another Registered Nurse notify Physician 3. Normal Saline at KVO unless hypotensive, then give Normal Saline $500 \mathrm{~mL}$ bolus 4 . Diphenhydramine $25 \mathrm{mg}$ IVP $\times 1$, then repeat every 3 hours prn for symptoms above 5. Oxygen at 2 Liters via nasal canulae 6 . Diphenhydramine $50 \mathrm{mg}$ IVP $\times 17$. Hydrocortisone $100 \mathrm{mg}$ IVP $\times 18$. Vital signs every 5 minutes until back to baseline, then every 15 minutes until resolutions of symptoms 9 . When symptoms resolve, resume infusion at $50 \%$ of the original rate 10 . Complete ADR report.

[X] Treatment Conditions 3 SEVERE REACTIONS: (Bronchospasm, stridor, wheezing, respiratory depression, cardiac arrythmia, generalized urticaria, SBP below $80 \mathrm{mmHG}$, angioedema, shock, loss of consciousness) IF PATIENT DEVELOPS RIGORS ANDIOR CHILLS CONTACT MD IMMEDIATELY FOR ORDERS, CONSIDER DEMEROL $25 \mathrm{mg} I \mathrm{M} \times 1,1$. Stop infusion and notify Physician 2. Normal Saline at KVO unless hypotensive, then give Normal Saline $500 \mathrm{~mL}$ bolus 3 . Oxygen at 2 Litres via nasal canulae 4 . Diphenhydramine $50 \mathrm{mg}$ IVP $\times 15$. Hydrocortisone $100 \mathrm{mg}$ IVP $\times 16$. Epinephrine $0.3 \mathrm{mg}$ IV $\times 1$, may repeat in 20 minutes $\times 1$ dose 7. Vital signs every 5 minutes until back to baseline, then every 15 minutes until resolution of symptoms 8 . For shortness of breath and chest tightness, use continuous oximetry 9. Complete ADR report.

[X] Nasal cannula oxygen Routine, UNTIL DISCONTINUED, Starting S For Until specified

Liters per minute: 2 LPM

FOR MODERATE and SEVERE REACTIONS

[X] Nursing Order: obtain patient Routine, ONE TIME For 1 Occurrences

consent and provide fact

sheet for patient/caregiver

prior to administration

11 Other

Figure 3. COVID-19 monoclonal antibody order set.

(C) 2021 Epic Systems Corporation. 


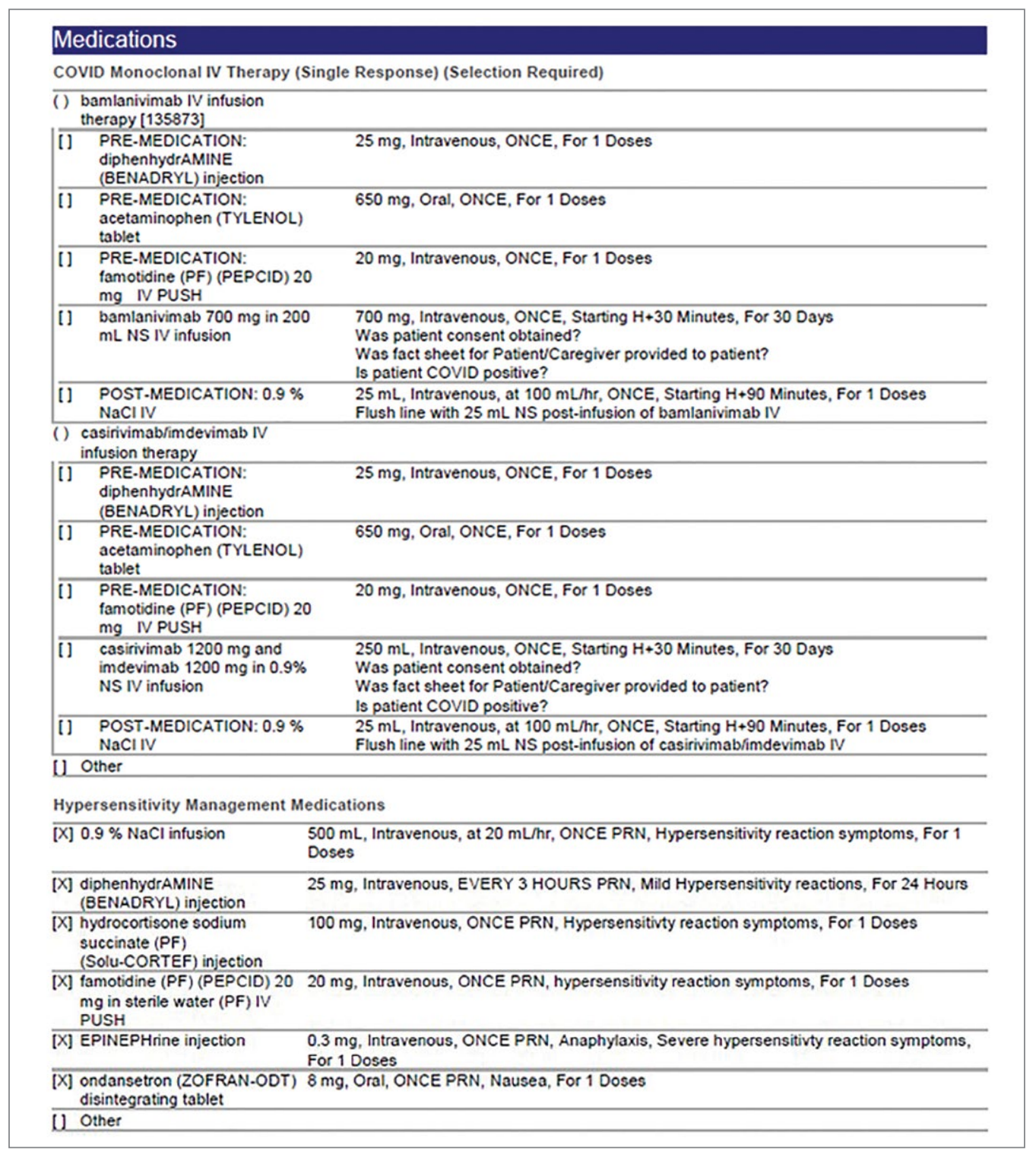

Figure 3. (continued)

the positive COVID-19 PCR test result is confirmed. If the patient no longer meets criteria, the patient is triaged accordingly, including evaluation for higher-level care (eg, supplemental oxygen, hospital admission). The second optoion is via new patient walk-ins without outpatient physician referral (Figure 4). In these cases, an initial screening is performed, documenting EUA criteria for use in the preliminary assessment (Figure 5). Physicians will consider an outside COVID-19 test as valid, so long as documentation is readily available confirming a positive PCR result.
Otherwise, an in-house COVID-19 PCR test will be performed, which has a 2-hour turnaround time.

Infusion Schedule. The ED offers a total of 16 COVID-19 monoclonal infusions slots daily. These are broken up into 4 infusion time blocks (eg, 8 AM, 12 PM, 4 PM, 8 PM), with each infusion time block consisting of 4 available patient appointments. A list of scheduled infusions for the day is emailed to the pharmacy department every morning, and patients are instructed to arrive 1 hour prior to their appointment time. This 


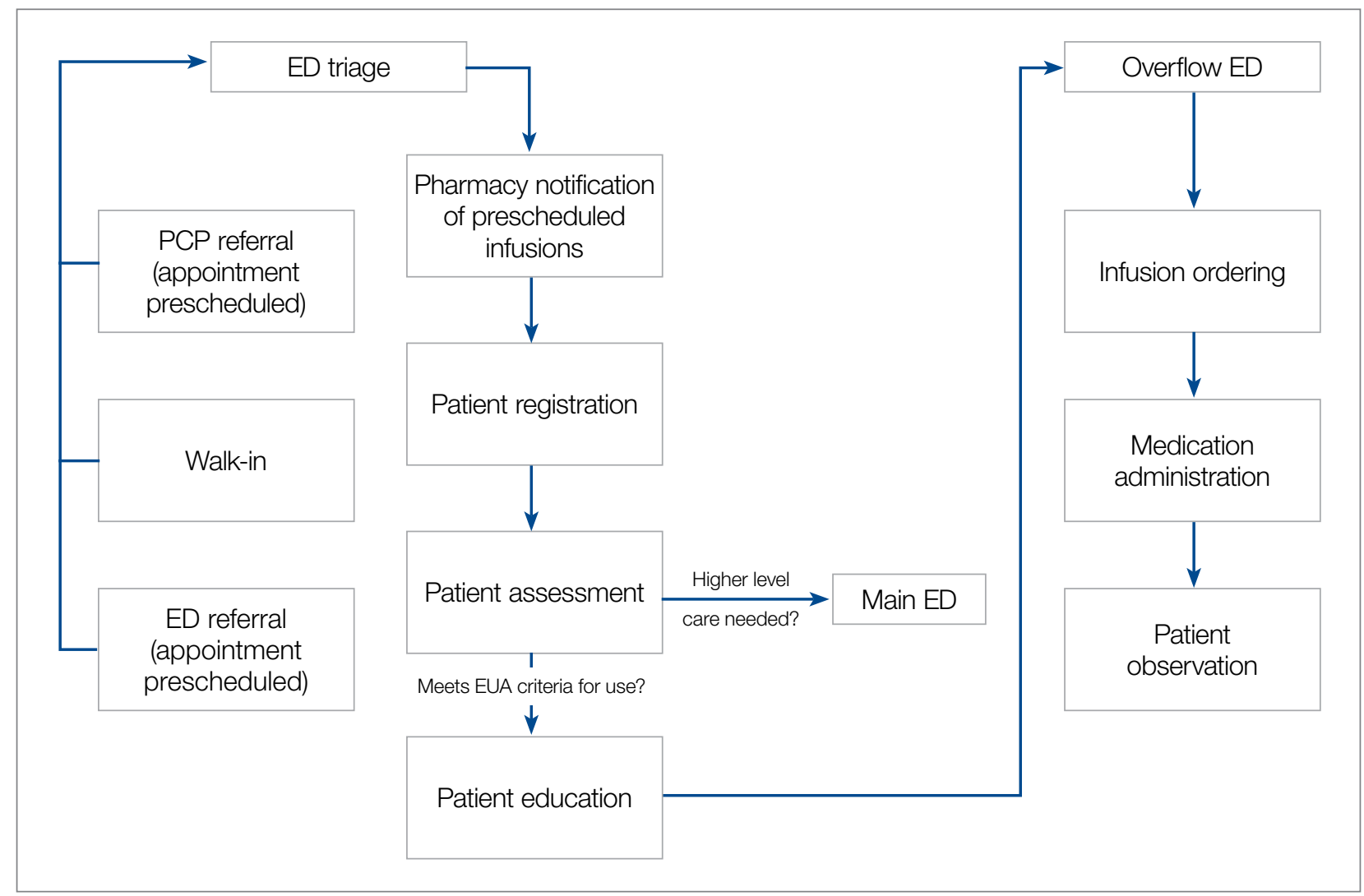

Figure 4. Workflow for COVID-19 monoclonal antibody infusion. This graphic depicts how patients arrive to Mount Sinai Medical Center's emergency department (ED) for COVID-19 monoclonal antibody infusion and the associated workflow. Patients are referred from their primary care provider (PCP), from an ED on a previous visit, or as a walk-in. In the triage area, the patient's eligibility is assessed. If it is determined the patient requires higher-level of care, the patient is triaged to a room within our main ED. If it is determined that the patient is a candidate for COVID-19 monoclonal antibody therapy, the patient is escorted to the overflow ED, which has become a dedicated station within the ED for treatment, administration, and observation.

allows time for patient registration, assessment, and pharmacy notification in advance of order entry. For logistical purposes, and as a patient safety initiative to reduce the likelihood of medication errors, each of the available COVID-19 monoclonal antibodies is offered on a designated day. Bamlanivimab is offered on Tuesday, Thursday, Saturday, and Sunday, while casirivimab/ imdevimab is offered Monday, Wednesday, and Friday. This provides flexibility to adjust should supply deviate based on Department of Health allocation or should new therapy options within this class of medication become available.

Patient Education. Prior to administration of the monoclonal therapy, physician and nursing staff obtain a formal, written patient consent for therapy and provide patients with the option of participating in the institutional review board (IRB) approved study. Details of this are discussed in the risk management and IRB sections of the article. Nursing staff also provides the medication-specific Fact Sheet for Patients and Caregivers in either Spanish or English, which is also included as a hyperlink on the COVID-19 Monoclonal Antibody Order Set for ease of access. Interpreter services are available for patients who speak other languages. An ED decentralized pharmacist is also available onsite Monday through Friday from 12 PM to 8:30 PM to supplement education and serve as a resource for any questions.

Infusion Ordering. Once the patient is ready to begin therapy, the he/she is brought to a dedicated overflow area of the ED. There are few, if any, patients in 


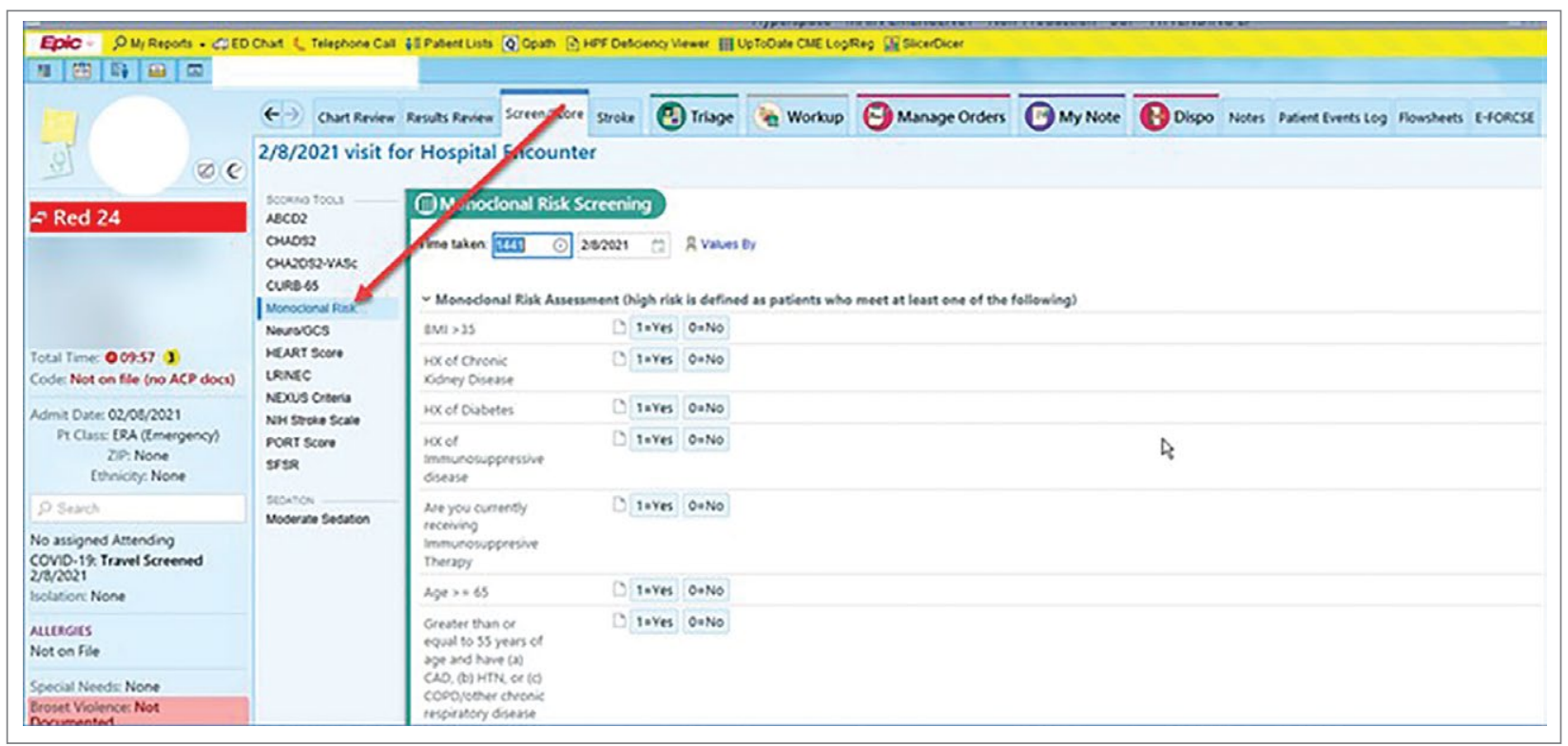

Figure 5. Electronic health record preliminary assessment. (C) 2021 Epic Systems Corporation.

this location, and it is adjacent to the main emergency center for easy access by the patients, nurses, pharmacists, and physicians. The physician then enters orders in the EHR using the COVID-19 Monoclonal Antibody Order Set (Figure 3). Three discrete questions were built into the medication order: (1) Was patient consent obtained? (2) Was the Fact Sheet for Patient/Caregiver provided to the patient? (3) Is the patient COVID-19 PCR-positive? These questions were built as hard stops so that the medication orders cannot be placed without a response. This serves as another double-check to ensure processes are followed and helps facilitate timely verification by the pharmacist.

Medication Administration. One nurse is dedicated to administering the monoclonal therapies scheduled at $8 \mathrm{AM}$ and $12 \mathrm{PM}$ and another at $4 \mathrm{PM}$ and $8 \mathrm{PM}$. Each appointment block is 4 hours in duration to allow adequate time for patient registration, infusion, and postinfusion observation. The nurse administers the premedications and COVID-19 monoclonal therapy, and observes the patient for the required 1-hour postadministration observation period. Nursing orders detailing monitoring parameters for mild, moderate, and severe reactions, along with associated medication orders to administer in the event they occur, are detailed in the nursing orders of the COVID-19 Monoclonal Antibody Order Set (Figure 3). Prior to administration, the nurse scans each medication and the patient's wrist identification band, and documents the time of administration within the EHR medication administration report.

\section{Pharmacy Department}

Medication Receipt Process. Inventory is currently allocated biweekly from the state department of health and will soon be transitioning to a direct order system. The pharmacy technician in charge of deliveries notifies the pharmacy Antimicrobial Stewardship Program (ASP) clinical coordinator upon receipt of the monoclonal therapies. Bamlanivimab is supplied as 1 vial per dose, whereas casirivimab/imdevimab is supplied as 4 vials or 8 vials per dose, depending how it is shipped. To reduce the likelihood of medication errors, the ASP clinical coordinator assembles each of the casirivimab/imdevimab vials into kits, where 1 kit equals 1 dose. Labels are then affixed to each kit indicating the medication name, number of vials which equal a full dose, and pharmacist signature. The kits are stored in a dedicated refrigerator, and inventory logs are affixed to the outside of the refrig- 
erator and updated daily. This inventory is also communicated daily to ED physician, nursing, and pharmacy leadership, as well as the director of patient safety, who reports weekly usage to the state Department of Health and Human Services. These weekly reports are used to determine allocation amounts.

Medication Verification and Delivery. The Mount Sinai Medical Center pharmacist staffing model consists of centralized order entry and specialized, decentralized positions. All orders are verified by the ED pharmacist when scheduled (not a 24/7 service) and by the designated pharmacist for all other times. At the time of medication verification, the pharmacist documents patient-specific EUA criteria for use and confirms that consent was obtained and the Fact Sheet for Patients/Caregivers was provided. A pharmacist intervention was developed to assist with this documentation. Pharmacists input smart text ".COVIDmonoclonal" and a drop-down menu of EUA criteria for use appears. The pharmacist reviews the patient care notes and medication order question responses to ascertain this information, contacting the ED prescriber if further clarification is required. This verification serves as another check to ensure processes put in place are followed. Lastly, intravenous preparation and delivery are electronically recorded in the EHR, and the medications require nursing signature at the time of delivery to ensure a formal chain of custody.

\section{Risk Management}

At Mount Sinai Medical Center, all EUA and investigational therapies require patient consent. Consistent with this requirement, a COVID-19 monoclonal specific consent was developed by risk management. This is provided to every patient receiving a COVID-19 monoclonal infusion, in addition to the FDA EUA Fact Sheet for Patients and Caregivers, and documented as part of their EHR. The questions providers must answer are built into the order set to ensure this process is followed and these patient safety checks are incorporated into the workflow.

\section{Billing and Finance Department}

In alignment with Mount Sinai Medical Center's mission to provide high-quality health care to its diverse commu- nity through teaching, research, charity care, and financial responsibility, it was determined that this therapy would be provided to all patients regardless of insurance type, including those who are uninsured. The billing and finance department was consulted prior to this service being offered, to provide patients with accurate and pertinent information. The billing and finance department provided guidance on how to document patient encounters at time of registration to facilitate appropriate billing. At this time, the medication is free of charge, but nonmedication-related ED fees apply. This is explained to patients so there is a clear understanding prior to booking their appointment.

\section{Infection Prevention}

As patients receiving COVID-19 monoclonal therapies can transmit the virus to others, measures to ensure protection for other patients and staff are vital. To minimize exposure, specific nursing and physician staff from the ED are assigned to the treatment of these patients, and patients receive infusions and postobservation monitoring in a designated wing of the ED. Additionally, all staff who interact with these patients are required to don full personal protective equipment. This includes not only physicians and nurses but all specialties such as physician assistants, nurse practitioners, pharmacists, and laboratory technicians. Moreover, patients are not permitted to go home in a ride share and are counseled on Centers for Disease Control and Prevention quarantining following infusion.

\section{Measurement of Process and Outcomes and Reporting}

IRB approval was sought and obtained early during initiation of this service, allowing study consent to be offered to patients at the time general consent was obtained, which maximized patient recruitment and streamlined workflow. The study is a prospective observational research study to determine the impact of administration of COVID-19 monoclonal antibody therapy on length of symptoms, chronic illness, and rate of hospitalization. Most patients were eager to participate and offer their assistance to the scientific community during this pandemic. 


\section{Staff Education}

In order to successfully implement this multidisciplinary EUA treatment option, comprehensive staff education was paramount after the workflow was developed. Prior to the first day of infusions, nurses, and pharmacists were provided education during multiple huddle announcements. The pharmacy team also provided screen captures via email to the pharmacists so they could become familiar with the order set, intervention documentation, and location of the preliminary assessment of EUA criteria for use at the time of order verification. The emergency medicine department chair and chief medical officer also provided education via several virtual meetings and email to referring physicians (specialists and primary care) and residents in the emergency centers involved in COVID-19 monoclonal therapy-related patient care.

\section{Factors Contributing to Success}

We believe the reasons for continued success of this process are multifactorial and include the following key elements. Multidisciplinary planning, which included decision makers and all stakeholders, began at the time the idea was conceived. This allowed quick implementation of this service by efficiently navigating barriers to engaging impacted staff early on. Throughout this process, the authors set realistic step-wise goals. While navigating through the many details to implementation described, we also kept in mind the big picture, which was to provide this potentially lifesaving therapy to as many qualifying members of our community as possible. This included being flexible with the process and adapting when needed to achieve this ultimate goal. A focus on safety remained a priority to minimize possible errors and enhance patient and staff satisfaction. The optimization of the EHR streamlined workflow, provided point-of-care resources, and enhanced patient safety. Additionally, the target date set for implementation allowed staff and department leads adequate time to plan for and anticipate the changes. Serving only 1 patient on the first day allowed time for staff to experience this new process hands-on and provided opportunity for focused education. This team communication was essential to implementing this project, including staff training of processes and procedures prior to go-live. Early incorporation of IRB approval allowed the experience to be assessed and considered for contribution to the scientific literature to tackle this novel virus that has impacted our communities locally, nationally, and abroad. Moreover, continued measurement and reporting on a regular basis leads to performance improvement. The process outlined here can be adapted to incorporate other new therapies in the future, such as the recent February 9, 2021, EUA of the COVID19 monoclonal antibody combination bamlanivimab and etesevimab. $^{10}$

\section{Conclusion}

We administered 790 COVID-19 monoclonal antibody infusions between November 20, 2020 and March 5, 2021. Steps to minimize the likelihood of hypersensitivity reactions were implemented, and a low incidence $(<1 \%)$ has been observed. There has been no incidence of infection, concern from staff about infection prevention, or risk of infection during the processes. There have been very infrequent cost-related concerns raised by patients, typically due to incomplete communication regarding billing prior to the infusion. To address these issues, staff education has been provided to enhance patient instruction on this topic. The program has provided patient and family satisfaction, as well nursing, physician, pharmacist, clinical staff, and hospital administration pride and gratification. Setting up a new program to provide a 4-hour patient encounter to infuse therapy to high-risk patients with COVID-19 requires commitment and effort. This article describes the experience, ideas, and formula others may consider using to set up such a program. Through networking and formal phone calls and meetings about monoclonal antibody therapy, we have heard about other institutions who have not been able to institute this program due to various barriers to implementation. We hope our experience serves as a resource for others to provide this therapy to their patients and expand access in an effort to mitigate COVID-19 consequences and cases affecting our communities.

Corresponding Author: Kathleen Jodoin, PharmD, BCPS, Mount Sinai Medical Center, 4300 Alton Rd, Miami Beach, FL 33140; kathleen.jodoin@msmc.com.

Financial Disclosures: None. 


\section{References}

1. COVID Data Tracker. Center for Disease Control and Prevention. https://covid.cdc.gov/covid-data-tracker/\#global-counts-rates. Accessed March 12, 2021.

2. Fact Sheet for Health Care Providers Emergency Use Authorization (EUA) of Bamlanivimab. US Food and Drug Administration. Updated February 2021. Accessed March 9, 2021. https://www.fda.gov/ media/143603/download

3. Coronavirus (COVID-19) Update: FDA Authorizes Monoclonal Antibodies for Treatment of COVID-19 | FDA. https://www.fda. gov/news-events/press-announcements/coronavirus-covid-19-update-fda-authorizes-monoclonal-antibodies-treatment-covid-19. Accessed February 14, 2021.

4. Fact Sheet for Health Care Providers Emergency Use Authorization (EUA) of Casirivimab and Imdevimab. US Food and Drug Administration. Updated December 2020. Accessed March 9, 2021. https://www.fda.gov/media/143892/download

5. Chen P, Nirula A, Heller B, et al. SARS-CoV-2 Neutralizing antibody LY-CoV555 in outpatients with COVID-19. N Engl J Med. 2021;384(3):229-237. doi:10.1056/NEJMoa2029849
6. Gottlieb RL, Nirula A, Chen P, et al. Effect of bamlanivimab as monotherapy or in combination with etesevimab on viral load in patients with mild to moderate COVID-19: a randomized clinical trial. JAMA. 2021;325(7):632-644. doi:10.1001/ jama.2021.0202

7. Weinreich DM, Sivapalasingam S, Norton T, et al. REGN-COV2, a neutralizing antibody cocktail, in outpatients with COVID-19. N Engl J Med. 2021;384:238-251. doi:10.1056/nejmoa2035002

8. Mulangu S, Dodd LE, Davey RT Jr, et al. A randomized, controlled trial of Ebola virus disease therapeutics. N Engl J Med. 2019;381:22932303. doi:10.1056/NEJMoa1910993

9. Boyle, P. Can an experimental treatment keep COVID-19 patients out of hospitals? Association of American Medical Colleges. January 29, 2021. Accessed March 9, 2021. https://www.aamc.org/news-insights/ can-experimental-treatment-keep-covid-19-patients-out-hospitals

10. Fact Sheet for Health Care Providers Emergency Use Authorization (EUA) of Bamlanivimab and Etesevimab. US Food and Drug Administration. Updated February 2021. Accessed March 9, 2021. https://www.fda.gov/media/145802/ download

\section{CALL for REVIEWERS}

The Journal of Clinical Outcomes Management welcomes applications from physicians, directors of pharmacy, nurse leaders, and others working in ambulatory, inpatient, and long-term care to serve as peer reviewers.

\section{You will not be asked to review more than 3 times per year.}

The following medical specialties are especially of interest: cardiology, critical care, endocrinology, hematology/oncology, hospital medicine, infectious diseases, mental health, neurology, nephrology, pulmonary disease, and rheumatology.

To apply to be a peer reviewer, email your CV along with a description of your subject areas of interest to eseger@mdedge.com.

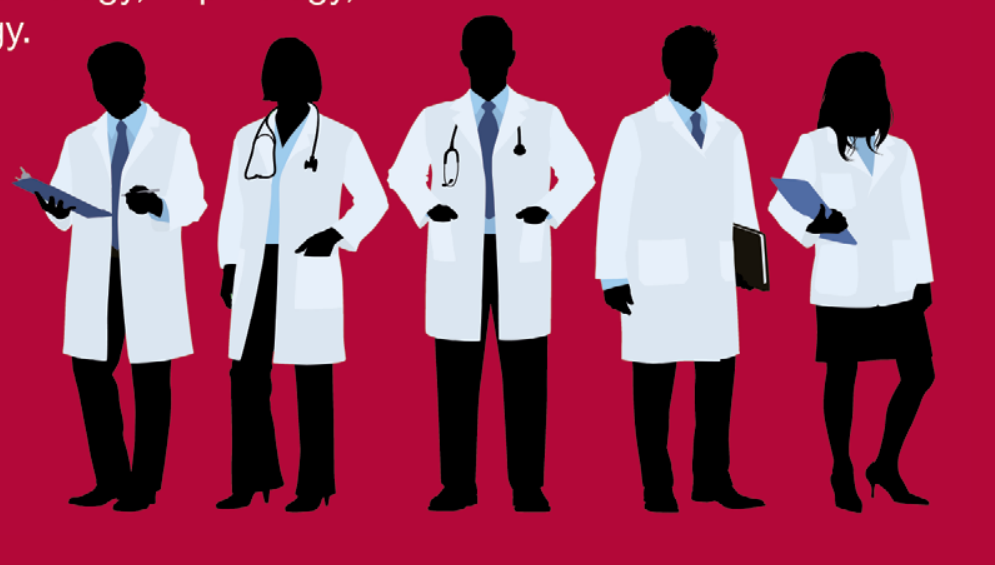

